\title{
Physical separation technology to support the strategic development of urban mining
}

\author{
— Development of unused/hard-to-use resources and a future vision \\ of resources for Japan -
}

Tatsuya OKI

[Translation from Synthesiology, Vol.6, No.4, p.238-245 (2013)]

\begin{abstract}
Most of the natural metal resources supporting manufacturing in Japan are imported from foreign countries. In recent years, the need for a stable supply has become a serious ongoing concern in the face of sudden price increases and unfavorable export regulations. Urban mining offers a promising means of utilizing domestic resources in order to minimize this risk. However, because the degree of integration of metals, including rare metals, in manufactured products is not necessarily high, the use of physical separation technology which can concentrate such metals and minimize costs is indispensable. Especially given the lack of experience in recovering certain types of rare metal elements from waste products, innovative separation technologies are needed. In this report, I introduce techniques for achieving rare metal recycling through innovations in physical separation, and present a plan for 'strategic urban mining,' based on physical separation technology and aimed at domestic circulation of metal resources.
\end{abstract}

Keywords : Urban mine, recycling, rare metal, physical separation, tantalum, strategic urban mining

\section{Introduction}

Japan is dependent on foreign imports for almost all its metal resources required for manufacturing. Recently, stable supplies have been jeopardized due to sudden increases in rare metal prices and/or changes in export regulations. Since the various metal resource elements have distinctive functions, unlike energy resources, each is difficult to replace with other metals. Therefore, even if the amount used is small, products utilizing such metals cannot be produced in Japan as long as the supply of these metals is interrupted; and over the long-term, this may threaten the foundation of Japan, a nation based on technology. When a manufactured product reaches the end of its lifespan, its contained metal elements remain unchanged; and thus in principle can be recovered as original raw materials. This means that horizontal recycling is possible. There are many waste products in Japan, and these are expected to become useful resources; thus the term 'urban mine,' coined by Dr. Michio Nanjo of Tohoku University in the 1980s, has been revived. However, such waste products are scattered throughout Japan, and the concentration of metal contained in them is often significantly less than in natural mines. To develop such hard-to-process resources, it is necessary to apply physical separation technology that enables the primary concentration of metals at a low cost. However, rare metals, in fact, have yet to be extracted as individual elements from waste products, and thus physical separation technology based on a new way of thinking is necessary. In this report, I shall describe a technique for metal recycling through innovations in physical separation, and initiatives to construct 'strategic urban mines' which form the foundation for domestic metal resource recycling.

\section{Physical separation technology for developing hard-to-process resources}

\subsection{Importance of physical separation technology in the development of urban mines}

Although there are very few metal mines currently operating in Japan, imported metal materials exist throughout the country, in the form of waste manufactured products. Such waste products are now referred to as 'urban mines.' However, for a mine to be properly functional, it must be economically feasible to extract the respective resources; thus, the mere existence of such products throughout the country does not, in itself, constitute a proper 'mine,' even in an extended sense. Moreover, whereas natural mines mark localized concentrations of terrestrial resources over a long period of time, such concentrations do not occur 'naturally' in the case of waste products with significant metal content. This means that 'urban mines' do not possess such localized existence, but rather must be identified and artificially 'concentrated' through intentional human engagement in urban mining, which, in technological terms, refers to techniques for minimizing the energy required for such artificial concentration.

Research Institute for Environmental Management Technology, AIST Tsukuba West, 16-1 Onogawa, Tsukuba 305-8569, Japan E-mail: t-oki@aist.go.jp

Original manuscript received January 20, 2013, Revisions received July 9, 2013, Accepted July 9, 2013 
Initially (in the -1980s-'90s), Japan's recycling priority was on the 'achievement of quantity' through the construction of a social system involving the collection of waste products. After the legal reform in the 1990s, recycling infrastructure for large home appliances was established, and when rare metals recycling began (2008-09), many thought rare metals could be recovered easily once the waste products were collected. However, in practice, such metals could not be extracted from the waste products at existing recycling facilities. At the same time, in the 1990s, shortage of landfill space became a social issue, and due to environmental restrictions, 'recycling of quantity' was the main concern. Therefore, iron, aluminum, plastics, and other materials found in large quantities were recycled to reduce the landfill volume; and with respect to the recovery of small home appliances, which had been conducted in designated regions from 2008, the target shifted to rare metals. However, because the concentration of certain rare metals is typically several hundred to several thousand ppm, 'recycling of quality,' based on the concept of limited resources, is necessary; attempting to recover rare metals from small appliances using conventional recycling infrastructure is similar to performing surgery with a pickaxe. Thus, by around 2010, awareness of the need for technological conversion from recycling of quantity to recycling of quality had emerged.

Rare metals are typically used in small quantities in manufactured products, and are thus found in lower concentrations than structural materials such as iron or aluminum. Therefore, economically feasible recovery through hydrometallurgical methods employing chemical reagents is difficult. In pyrometallurgy, involving high-temperature reactions, while it is possible to efficiently recover copper and precious metals even at low concentrations, the recovery of rare metals is technologically difficult since many of these metals melt and disperse in the glasslike slag. To recover rare metals from waste products, it is essential to separate them from copper and precious metals, through physical separation, before smelting.

In addition, the precise meaning of 'rare metal' is not universally agreed upon. In Japan, 47 elements, including 17 rare earth elements, were designated as rare metals in the 1980s. Alternatively, rare earth is considered to be one type, and reference is made to 31 mineral types. Many countries, in fact, do not use the term 'rare metal,' and if they do, the definition may vary. Recently, the English-speaking world has adopted the term 'rare metal,' as used in Japan. However, questions have been raised about the utility of lumping everything into one group since, for example, platinum and palladium are rare metals, while gold and silver are not, though all are precious metals. Therefore, this study employs the term 'strategic metals' to describe metal resources that are strategically significant for Japan.

\subsection{Technological issues in physical separation}

Although individual issues are involved for each type of waste product, we will here consider the common fundamental issues involved in the preliminary physical separation of rare metals and copper/precious metals. Waste products typically consist of composite particles which are broken apart in the process of 'crushing.' The effects of crushing in many industrial processes include improvement in mobility, processability, and reactivity; and the objective of crushing is to obtain 'uniformity' of the resulting powder. On the other hand, the sole objective of crushing in physical separation is to promote liberation. Liberation is the creation or condition of a state in which each particle is composed of only a single substance, allowing us to designate a specific 'ingredient' as the target of the recovery, the element, alloy, or part that one wishes to recover. Therefore, crushing is positioned as a pretreatment in which the 'non-uniformity' of the powder is prepared for liberation. Since physical separation is the process in which the individual particles are sorted, the particles must be liberated before this process, or no advanced separation can be achieved, regardless of the technology employed. Figure 1 shows the relationship between the progress of liberation through crushing and the uniformity of the powder, from the perspective of this study. When the composite particle (initial particle) is broken down by crushing, it becomes an aggregation of liberated particles composed of single ingredients, and the non-uniform condition is achieved as the compositions of the individual domains become distinct. The ideal is to achieve this state in the stage of coarse (large) particles (achieving the 'mixed nuts' state as a result of crushing), since, if further crushing is necessary or the liberation can only be achieved through fine crushing, the uniformity of the composite (state of being well mixed) increases, and it becomes difficult to recover specific particles through separation (similar to attempting to separate the ingredients of mixed spices). The promotion of liberation by crushing sacrifices non-uniformity of the aggregate while achieving non-uniformity of the individual particle. Therefore, it is important to achieve liberation in the coarse-particle stage as much as possible, and excessive fine grinding must be avoided. Also, items whose crushing will result only in fragmentation without liberation (such as instant coffee) will not be useful targets for physical separation.

In the crushing process schematized in Fig. 1, it is possible to achieve good liberation through selective crushing, rather than 'random crushing' where the composite particles are equally crushed. Such a method, whereby liberation is achieved in the coarse-particle stage, is called 'selective crushing,' and this is one of the most important pretreatments for physical separation. However, since it is necessary to deal with diverse properties and structures in the target materials, selective crushing is technologically very difficult, and there is no device that serves as an all-purpose selective crusher. 
Currently, we must wait for suitable selective crushing functions for specific targets to be discovered by chance among various crushers; therefore, a systematic and logical organization of relevant technology is required.

Even if liberation occurs, various particles are typically still mixed after crushing, and separation will be necessary. In physical separation, differences in particle properties are exploited. Dry separation, conducted in a gas (usually air), can be done at low cost and with low environmental load, since drying and water treatment are not necessary. In contrast, wet separation, conducted in a liquid (normally water), though typically resulting in high separation precision, requires power for water circulation as well as drying, and is therefore disadvantageous in terms of energy and cost. In wet separation employing a large amount of surfactants to exploit particle surface properties, the load of wastewater treatment increases in comparison with methods exploiting particle bulk properties. For these reasons, dry separation is preferred in recycling, but there are limitations on the applicable particle size for each separation method, and the process cannot be completed through dry separation alone. Although it is difficult to delineate the minimum applicable particle size, it is roughly $1 \mathrm{~mm}$ for dry separation and roughly $50 \mu \mathrm{m}$ for wet separation using bulk properties, while anything below this will require wet separation using surface properties. Thus, energy and cost savings in physical separation primarily depend on the extent to which liberation can be achieved in the coarse-particle stage of the preliminary crushing process.
Figure 2 shows a model of the simplest physical separation process, consisting of one crushing and one separation process. The initial products are either a single type of waste product, an assortment of such products, or extracted printed circuit boards. There are various types of crushers, and the manner of crushing varies widely, depending on the conditions of the specific device and its operation. If liberation is achieved in the coarse-particle stage, through selective crushing by establishing optimal crushing conditions, the subsequent separation process can be performed with great efficiency. However, there are only a few conditions under which such selective crushing can be achieved, among the various combinations, and such selective crushing conditions have not been established for most waste products. In rare metal recycling, it is often the case that subsequent separation methods are sought without recognizing that the liberation of the crushed material is inadequate under the given conditions. The overall effect of the physical separation process is determined by the synergetic effect between the crushing and separation functions. Therefore, it is useless to deploy an excellent separator in the latter process without due consideration of the former process, and the initial key to success is the efficient achievement of liberation through selective crushing.

However, even if optimal liberation is achieved here, the particles remain in a mixed state; thus this process is properly understood only as a preparation for separation. As noted above, there are applicable particle sizes for

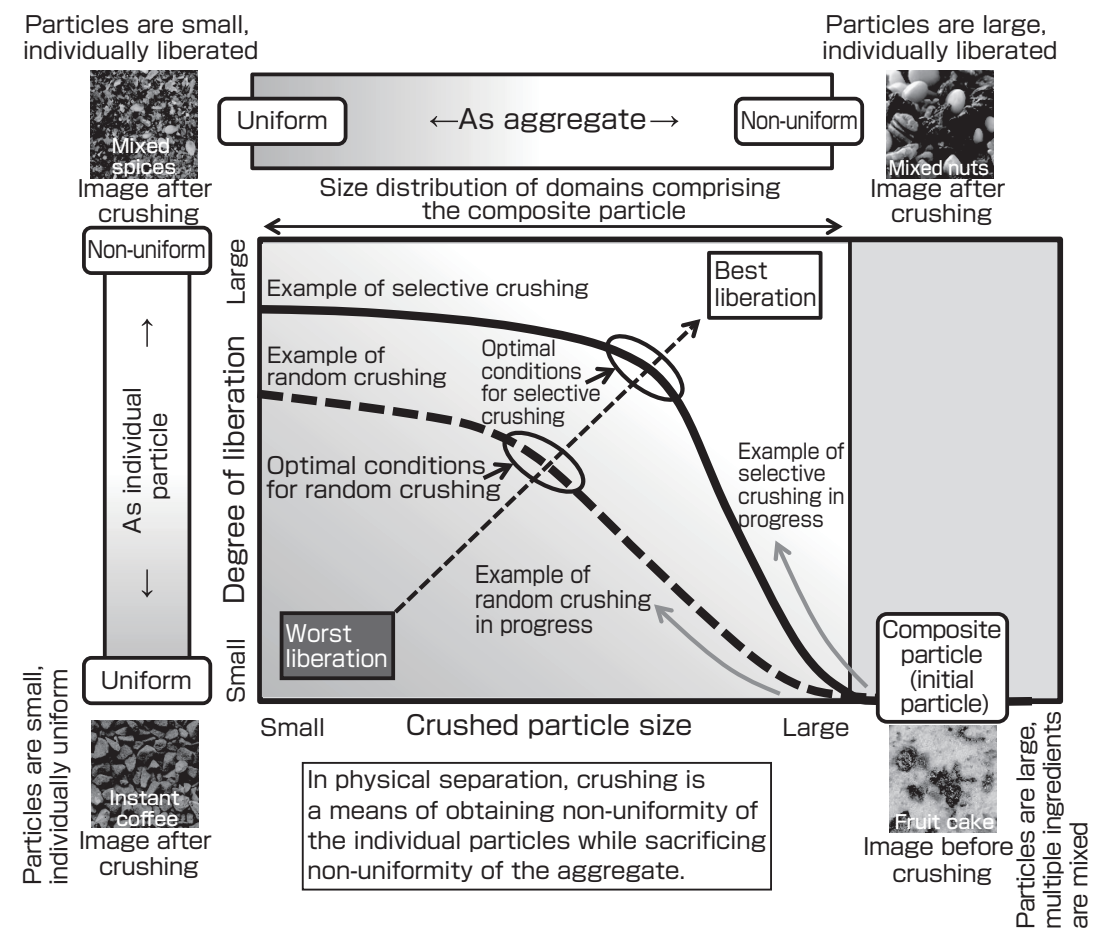

Fig. 1 Relationship between the progress of liberation through crushing and the non-uniformity of the powder 
each separation method, and even if dry separation can be applied, there are various types of devices for the various exploitable particle properties (size, shape, specific gravity, magnetism, conductivity, color, x-ray transmittance, etc.), and an even greater variety of separators available for a given property characteristic. Moreover, as in the case of the crushing process, the separation products may vary widely, depending on the conditions of the particles and the specific operation. In the simple, one crushing/one separation model shown in Fig. 2, there are seven parameters, each with tens of selections, and the overall patterns can number in the hundreds of billions. In practice, there will be two or three viable crushing processes and three to ten viable separation processes, and the number of patterns will be astronomical. However, the conditions for achieving optimal physical separation of hard-to-process resources from urban mines are limited to only a few patterns. Furthermore, in contrast to the lifespan of natural mines, from which ores with uniform composition can be mined for decades, the product cycle in urban mines is typically brief. Therefore, the viability period of a given separation pattern for a specific target is typically short, often only a few years, since the type, form, and quantity of rare metals in the respective waste products vary. Since, at the present time, it is difficult to identify the optimal conditions among such a vast number of possibilities in a reasonable time, processing is typically done under extremely unsatisfactory separation conditions.

\section{Achievement of recycling through innovations in physical separation}

\subsection{Alternative technology to manual dismantling and sorting - Easy sensing}

While it is difficult to determine the conditions for selective crushing that will achieve liberation, liberation can be accomplished through 'dismantling' individually if the size of the recovered object is relatively large. For example, the process of recovering the motor from large appliances is performed by hand, even in Japan, and it is not uncommon to recover target parts by hand sorting. Since waste products have complex structures and the mix of such products in recycling varies from day to day, manual dismantling and sorting are the most reliable methods for liberation and recovery. However, as there are limits on the application of this method in Japan due to considerations of economy, productivity, and international competitiveness, research into automation is underway. While there is, at present, no example of completely automated dismantling technology, various automated sorting technologies have been devised to replace traditional hand sorting. These, however, often involve expensive devices that deploy sophisticated sensing technology used in manufacturing processes; and while they are good at removing unwanted elements from almost uniform products, adequate performance may not be achieved in the case of dismantling waste products with numerous variations.

Therefore, the author has developed a low-cost, 'easy sensing' device, which automizes the dismantling and sorting of specific waste products. In this device, instead of expensive sensor technology, several inexpensive sensors, mimicking the five human senses, are combined to achieve

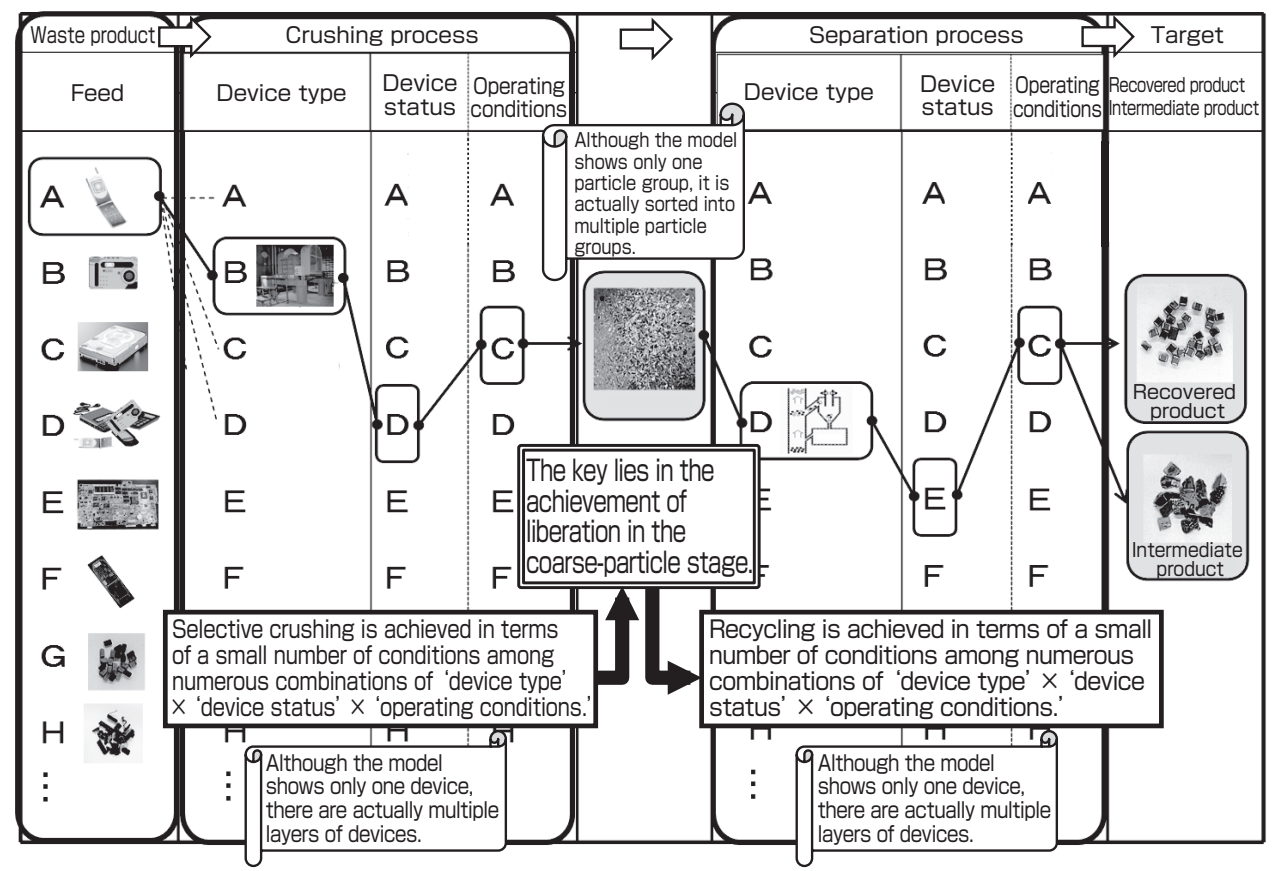

Fig. 2 Highlights of the process of optimization based on the physical separation (one crushing/one separation process) model 
high-precision separation, through manipulation based on respective product information. For example, the author has proposed a two-step crushing/separation method in which high-purity neodymium magnets containing rare earth are recovered from hard disk drives (HDDs). The 'HDD cutting separator (HDD-CS)' is one example used in this method. When a HDD is crushed, the powerful neodymium magnet typically adheres to the inside of the crusher, causing difficulties such as screen clogging. Even if it should fortunately be expelled from the crusher, a magnetic aggregate may immediately form on the nearby crushed steel plates, and liberation cannot be achieved. Therefore, the typical practice is to incorporate a demagnetizing process as part of the physical separation. Since the neodymium magnet has a low Curie temperature, its magnetism can be removed by heating to around $350^{\circ} \mathrm{C}$. However, since it is not efficient to heat 50 -times more metal in order to demagnetize the neodymium magnet comprising only about $2 \%$ of the total HDD weight, the search for an efficient demagnetization method continues. The author focused on the fact that HDDs, unlike other small appliances, have roughly the same shape and structure regardless of the manufacturer, year, or model. The HDD-CS (Fig. 3), developed jointly with Kinki Industrial Co. Ltd., senses the stray magnetic field of the HDD surface, using four magnetic sensors and two position sensors, and detects the position of the neodymium magnet using a nondestructive method. The HDD is then transported directly beneath the non-magnetic cutter, and the magnet part is punched out before demagnetization. A database for leakage magnetic flux density for each HDD is created, in order to increase the detection precision based on device optimization data. Although the device is small and inexpensive, it can automatically process 400,000 to a million HDDs per year. The magnetic parts, concentrated roughly tenfold by the device, undergo demagnetization, impact crushing, and screening, and magnet powder of $94-97 \%$ purity has been recovered.
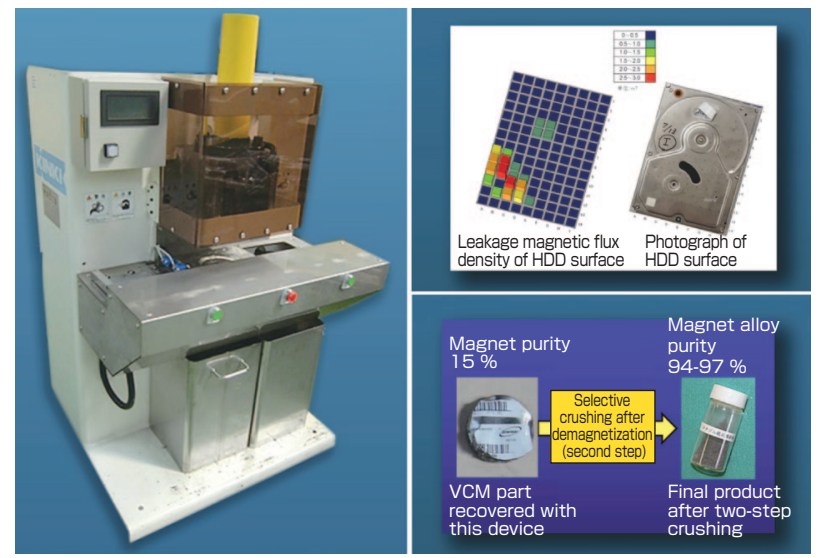

Jointly developed by author and Kinki Industrial CO. Ltd., license agreement with Kinki Industrial CO. Ltd.

Fig. 3 Recycling of neodymium magnets using an HDD cutting separator (jointly developed with Kinki Industrial Co. Ltd.)

\subsection{Automated control of separation - Smart operation} In the case of HDDs, we were able to recover the magnet merely through impact crushing and screening, but normally separation optimization is difficult even if liberation is achieved. As noted earlier, there are typically three to ten steps in a separation process, and the combinations of separation conditions are astronomical. In the face of such a massive number of possibilities, the typical response is to abandon the thought of device separation, before ever having sought to optimize the performance of the device. The author investigated a method for quickly obtaining such optimal conditions, through numerical computation using a database; and the resulting 'smart operation' system automatically operates the device under optimal conditions, without need of skilled worker involvement. By applying this technology to the recycling of printed circuit boards, we were able to develop the world's first separation process that enables successful recycling of tantalum capacitors; and in the spring of 2012 the system was installed in a recycling plant promoting the urban mining business model.

Tantalum is an expensive metal among rare metals. As it is rarely recycled at present, it has been designated by the Japanese government as one of the five mineral types (tungsten, tantalum, cobalt, neodymium, and dysprosium) to which recycling should be given priority. Most of the tantalum in use is employed in the tantalum capacitors on printed circuit boards. In the early stages of system development, the author followed the conventional separation theory (Fig. 1) and attempted liberation by finely grinding the printed circuit boards, focusing solely on the liberation of tantalum elements (which exist mainly as oxides). However, recovery was not possible after fine grinding and separating, because of tantalum's oxide properties. Although the tantalum could be concentrated several times, it could not be recovered since the weight ratio of tantalum in the printed circuit board was about $1,000 \mathrm{ppm}$, and it could only be recovered as a heavy product along with precious metals and other heavy metals. As noted above, rare metals such as tantalum must be separated from copper/precious metals before pyrometallurgical treatment, and thus tantalum could not be extracted as a resource using the above method. However, at a joint-research partner company, an electronic device was exfoliated from the printed circuit board in its original form using a particular crusher. Nonetheless, it seemed impossible to recover only certain electronic devices among the wide variety of such devices. However, the author reasoned that each electronic device, among this seemingly disorderly amalgam, would possess unique separation properties because all were artificial; and thus attempted to determine the optimal separation pattern in which the tantalum capacitor was targeted as the liberation item. It seemed impossible, however, to determine such optimal conditions based on conventional separation experiment data. Therefore, we categorized over 400,000 electronic devices, 
recovered from PCs, into 1,680 categories, and created a database containing their characteristics and separation properties. Then, roughly two quadrillion separation patterns, including repeated use, were generated through numerical computation, for three separation methods, including size, specific gravity, and magnetic separation, to narrow down the optimal conditions for concentration of the tantalum capacitors. By this means, separation conditions were discovered in which the recovery rate (weight percentage of recovered tantalum capacitors among the tantalum capacitors in the exfoliated electronic devices) was $80 \%$ or more, and the quality (weight percentage of tantalum capacitors in the recovered product) was $80 \%$ or more, in six steps, beginning with the separated electronic devices (Fig. 4).

Although the optimal separation pattern became apparent, there was no existing device that satisfied these separation conditions; and therefore dedicated devices were developed. The inclined and low-intensity magnetic-shape separator (Fig. 5a) is a small device that plays a supplementary role. It is a hybrid device that recovers aluminum electrolytic capacitors in the inclined conveyor separating area, and quartz resonators in the weak magnetic separating area. In the shape-separating area, the cylindrical aluminum electrolytic capacitors are recovered by rolling, and a constant load swing pin-gate mechanism was newly developed to enhance the rolling process. In conventional magnetic separators, some of the tantalum capacitors adhered to the magnet. Therefore, a mechanism for accurately maintaining an extremely weak and uniform magnetic flux density on the conveyor surface was developed, and this was successful in recovering only the quartz resonators that contained high amounts of iron. Using this device, iron and aluminum are recovered separately, and the supply volume is restrained by sending the remnant, including tantalum capacitors, into a pneumatic separator. This double-tube pneumatic separator (Fig. 5b) is the main device in the separation process. The air velocity inside the two tubes can be accurately controlled using one blower. In the first tube, electronic devices heavier than the tantalum capacitors are collected by gravity, while the tantalum capacitors and lighter devices are sent to the second tube, where only the tantalum capacitors are recovered, again by gravity (Fig. 5c). Based on numerical computation, the air velocity in the first tube is set slightly higher than that in the second tube. Normally, when updraft is generated in a tube, the velocity will be greater in the center than on the periphery. Given this, in the present system, even among similar electronic devices some will rise and others will fall, and precise separation cannot be expected. Therefore, the author developed a mechanism

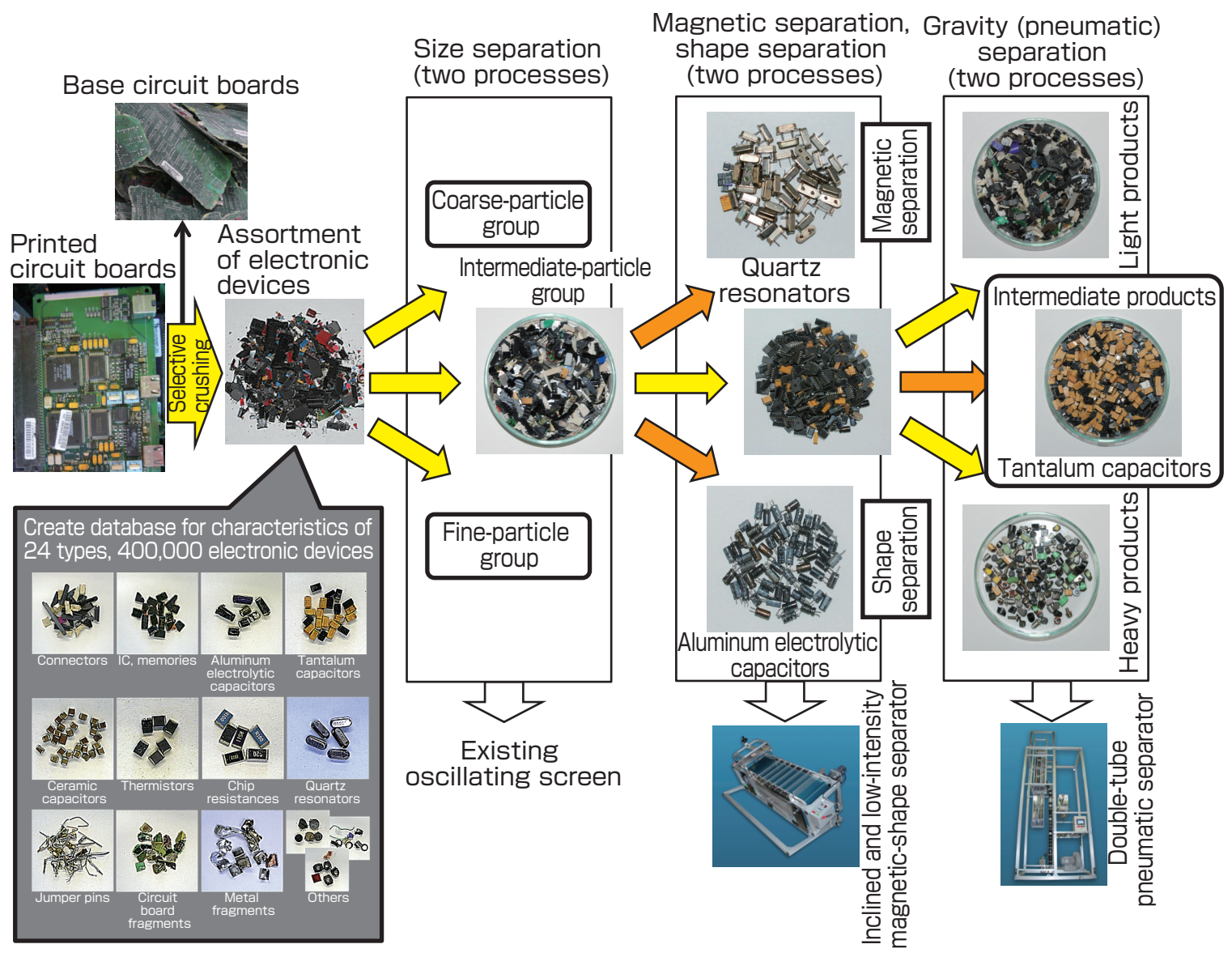

Fig. 4 Recovery process for tantalum capacitors extracted through calculation based on the database 
for creating uniform cross-sectional air velocity in the tube (Fig. 5d), which enables clear-cut separation. Also, devices with falling velocity near to that of the updraft inside the tube tend to fall into an irregular up-down motion, based on the posture of the devices in the tube, thereby impeding rapid separation. To avoid this, an orifice structure was installed to increase separation speed. Also incorporated, for the first time ever in such a device, were several mechanisms acting as pneumatic separators, including a joint mechanism whereby the devices travel quickly while the air velocity is held constant in the two tubes. Through operational control based on the electronic device database, the entire operation, from calibration to the recovery of devices, can be automated merely by selecting the target devices (such as the tantalum capacitor) on the display.

By means of these developments, the previous grade of recycled tantalum capacitors (limited to roughly 10-30\%), was dramatically improved, to a maximum of $97 \%$ quality, in the test run at a commercial plant in which the two separators were installed. Moreover, even if the target product specifications are partially changed, there is no need to start from scratch: recalculation of the optimal separation conditions can be easily performed simply by updating the respective product information in the database. Although installation of easy sensing and smart operation technology is only recently underway, it is expected that several hard-to-process resources will be efficiently recovered through these innovations in physical separation technology.

\subsection{Strategic Metal Resource Circulation Technology (Urban Mining) Project}

In urban mining, recycling cannot be achieved merely through the introduction of new technology in the resource recycling loop; the peripheral environment must also be restructured. To achieve sustainable cycling of strategic metals, it is important to construct not only appropriate resource processing technology such as physical separation devices, but a series of complementary systems, from product design to supply of recycled materials. Thus, the author initiated the Strategic Metal Resource Circulation Technology (Urban Mining) Project, whose aims extend from the technological development of strategic metal recovery from current urban mines to the creation of future strategic urban mines, supported by the strategic budget of AIST (the project was renamed the 'Strategic Metal Domestic Resource Circulation Project' in 2013). The project aims to promote AIST's resource circulation vision, and to organize and coordinate the research bases for strategic metal recycling in Japan, over a three year period beginning in FY 2012. This project consists of 5 topics that will be studied by over 30 AIST researchers across 7 research units (Fig. 6). Topic 1 is the selection of next-generation strategic metals and the evaluation of their true recycling potential. Topic 2 is the construction of a property and separation database for waste products that are targets for strategic metal recovery, and the development of automated separation technology for products containing significant quantities of strategic metals. Topic 3 is the establishment of pretreatment technology for smelting, to ensure that recovered waste products become

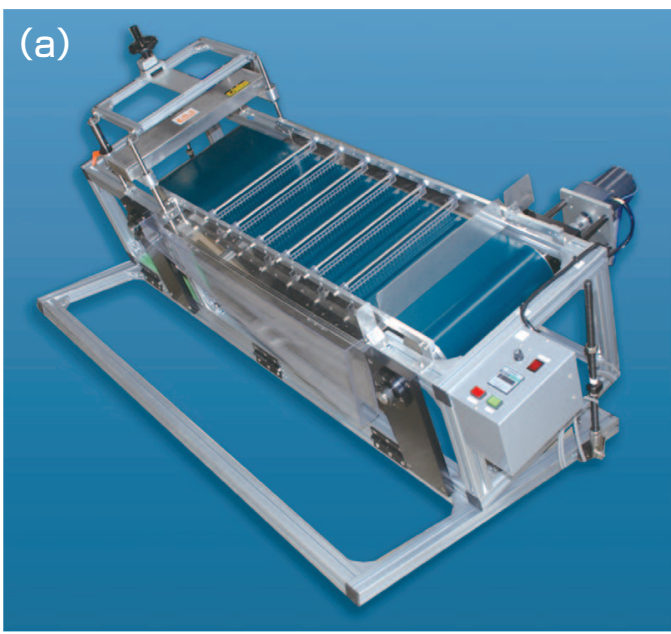

Developed by author, licensing agreement with Eriez Magnetics Japan Co., Ltd.
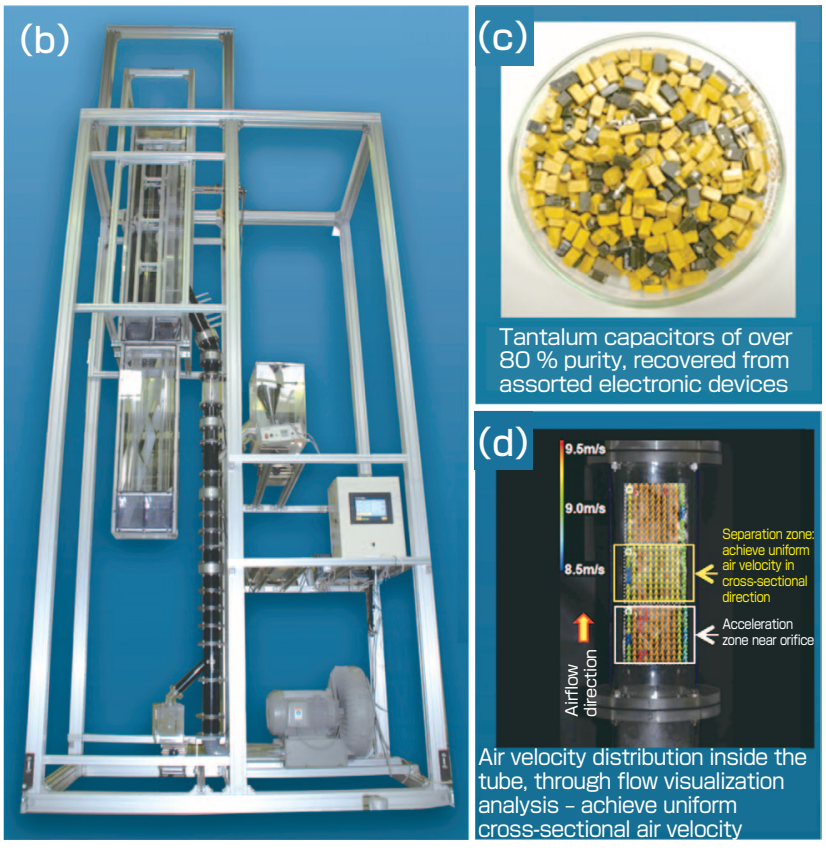

nalysis - achieve uniform

Developed by author, licensing agreement with Eriez Magnetics Japan Co., Ltd.

Fig. 5 Recycling of tantalum capacitors using inclined and low-intensity magnetic-shape separator and double-tube pneumatic separator 
appropriate smelting materials. Topics 1-3 advance the 'recycling vision' in which strategic metals are recovered from the current 'disorderly' urban mines. Topic 4 involves study of the necessary minimum recycling design guidelines to enable recycling of products that remain difficult to recycle even after the investigation in Topics 2 and 3, particularly from the perspective of physical separation technology. The proposal of a recycling 'production vision' in Topic 4 aims at the construction of 'strategic urban mines' whose future waste products will be suited to the Japanese recycling infrastructure from the production stage. Topic 5 advances AIST's 'production-recycling integrated vision,' which is a fusion of Topics 1-4. Highly efficient and internationally competitive resource circulation cannot be achieved as long as individual recycling technologies developed in Japan are scattered and disorganized. Therefore, the 'Strategic Urban Mining Research Base (SURE)' has been organized as a base for the industrialization of Japanese recycling technology, and it aims at full operation after the project's completion.

\section{Summary - Prospects for R\&D}

Japan has very few natural metal resources, and urban mines are one of its most promising domestic resources. Fortunately, it has utilized an abundance of rare metals in manufacturing and has focused on recycling in a timely fashion as a means of maintaining supplies. Therefore, as of 2013, the level of rare metal recycling technology in Japan is unparalleled in the world, exceeding that of Europe and the United States. If this advantage can be maintained in future, it can be exploited as a resource development tool comparable in value to the natural endowments of resourcerich countries. However, many challenges must be overcome. First, since it is difficult to forecast the future product market, it is difficult to design the recycling technologies that will be needed in, for example, 30 years; and even if such technology is designed, the period during which it can be employed will typically not be long because the product cycle is short. Furthermore, as the products are updated, resource savings and switchovers to less expensive metals will increase in the mid- to long-term. The concentration of important metals in products will gradually decrease, and further advanced recycling technologies will become necessary. On the other hand, though a stable supply of rare metals is of decisive importance to manufacturing, the distribution volume of the rare metals themselves is low and the market is not large. Therefore, they do not require large-scale recycling infrastructure, and new technologies developed by Japan are likely to be implemented relatively quickly in other countries. Thus, for Japanese technology to remain preeminent, it is necessary to maintain a technological advantage of three to five years at all times, and be able to swiftly and continuously introduce new recycling technologies.

In the development of urban mines, many issues center on physical separation. However, in the current situation, the comprehensive effort to tackle large issues and the development of new technologies are progressing sluggishly because the number of researchers in the field is small. Therefore, the development of urban mines may lag behind the social demand, even before encountering international competition. Thus, the Strategic Metal Resource Circulation Technology (Urban Mine) Project, in which strategic efforts, from production to the development of recycling technologies, are made in concert with corporate collaboration and human resource training; along with the resulting Strategic Urban Mining Research Base (SURE); are expected to provide visionary leadership with regard to resources in Japan, in order for the country to survive in the face of resource nationalism on the part of other countries.

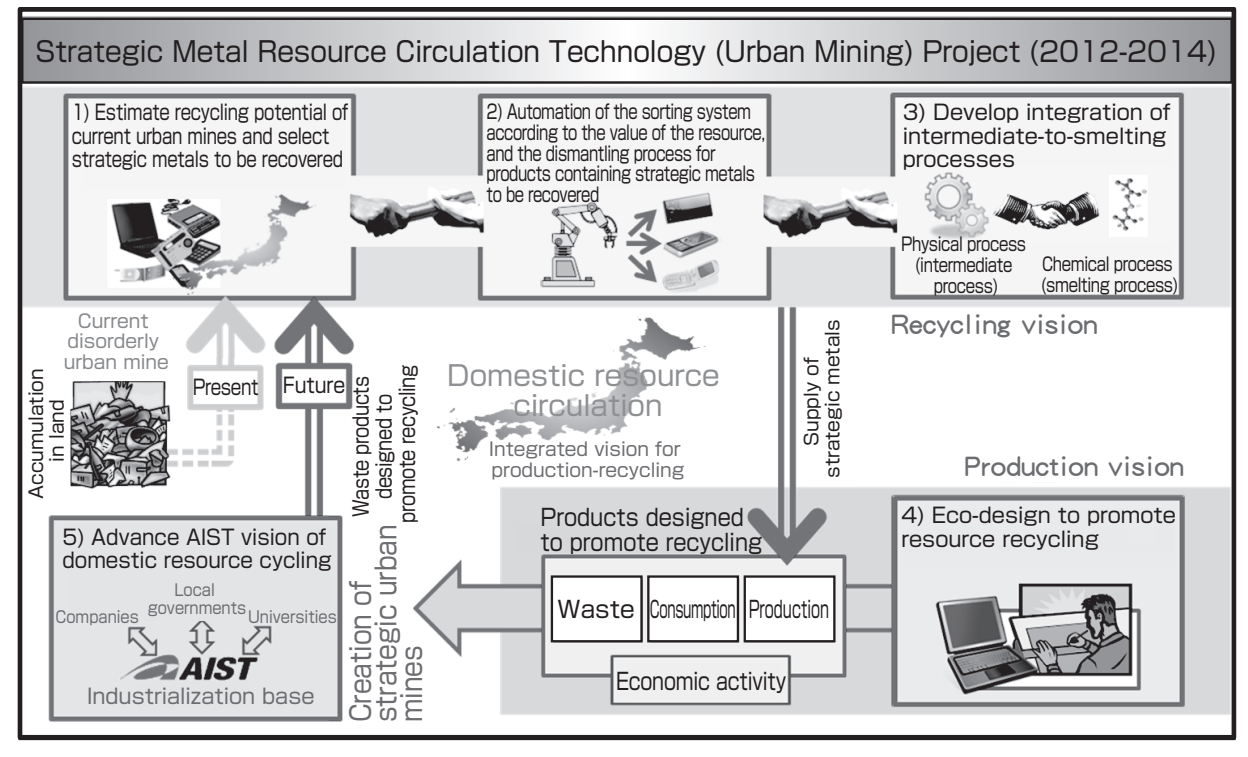

Fig. 6 Outline of Strategic Metal Resource Circulation Technology (Urban Mining) Projec 


\section{Author}

\section{Tatsuya OKI}

Completed the doctoral program at the Graduate School of Science and Engineering, Waseda University, in 1994. Ph.D. (Engineering). Worked as a research associate at Waseda University, and then joined the National Institute of Resources and Environment, part of the Agency of Industrial Science and Technology, of the Ministry of International Trade

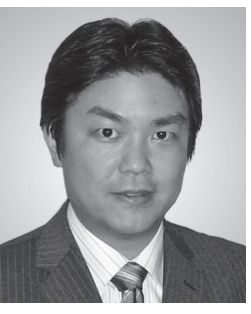
and Industry, in 1995. Leader of the Advanced Recycling Technology Research Group, of the Research Institute for Environmental Management Technology at AIST, in 2009. Engages in the development of recycling technology for rare metals, including tantalum capacitors, mineral processing technology for submarine hydrothermal polymetallic sulfide, and others. Participated in several NEDO and JOGMEC projects involving resource processing technology. Established the Strategic Urban Mining Research Base (SURE) at AIST in 2013.

\section{Discussions with Reviewers}

\section{Overall comment}

Comment (Yasuo Hasegawa, AIST; Mamoru Nakamura, Materials Research Institute for Sustainable Development, AIST)

This paper describes $R \& D$ in which rare metals are successfully recovered from waste home appliances through optimal physical separation; whereas, before, this technology was not viable due to cost. I think this research paper is appropriate for Synthesiology, both in composition and content. The proposal for the design of processing technology that allows "waste products" to be used as "urban mines" by efficiently extracting valuable rare metals from various discarded products, as well as the related testing process, are useful and will also be of value to researchers in other fields.
Question \& comment (Yasuo Hasegawa and Mamoru Nakamura)

Please explain in detail why rare metal recovery from waste home appliances was not successful until now, and the precise nature of the breakthrough for the author? Answer (Tatsuya Oki)

Rare metals account for only a very small fraction of the composition of most manufactured products, far below the concentration of structural materials such as iron and aluminum. Therefore, economically feasible recovery through pyrometallurgical methods involving high-temperature reactions, or hydrometallurgical methods involving chemical reagents, has not been possible. It is important to achieve primary concentration by means of physical separation. To this end, it is necessary to develop technology capable of liberating large particles as much as possible, or separating intact electronic devices from printed circuit boards, and to selectively recover the elements containing rare metals.

In the case of the study's tantalum capacitors, the characteristics and separation properties of 400,000 electronic devices recovered from PCs were entered into a database, and projections were made, through numerical computation, for the results of roughly two quadrillion separation patterns, including repeated use, for the size, specific gravity, and magnetic separation methods. We discovered separation conditions that enabled the recovery of tantalum capacitors at a rate of over $80 \%$ both in purity and recovery, in six steps, from the separated electronic devices. I think the greatest breakthrough consisted in determining the optimal separation conditions, and this process is described in subchapter 3.2.

The development of technology for recovering neodymium magnets containing rare earth from HDDs, described in subchapter 3.1 , involved the unique process of punching out the neodymium magnets that had been detected nondestructively by leakage magnetism using magnetic and position sensors. By concentrating the magnetic material roughly tenfold, significant energy savings and increases in economic efficiency were achieved in the subsequent demagnetization, impact crushing, and screening processes. In this technological development, the leakage magnetic flux densities of various HDDs were entered into the database; and, through optimization based on this data, automated processing of 400,000 to a million HDDs per year could be achieved by a small, low-cost device. 